PROCEEDINGS OF THE

AMERICAN MATHEMATICAL SOCIETY

Volume 134, Number 6, Pages 1561-1567

S 0002-9939(05)08450-9

Article electronically published on December 14, 2005

\title{
ON ROURKE'S EXTENSION OF GROUP PRESENTATIONS AND A CYCLIC VERSION OF THE ANDREWS-CURTIS CONJECTURE
}

\author{
S. V. IVANOV
}

(Communicated by Jonathan I. Hall)

\begin{abstract}
In 1979, Rourke proposed to extend the set of cyclically reduced defining words of a group presentation $\mathcal{P}$ by using operations of cyclic permutation, inversion and taking double products. He proved that iterations of these operations yield all cyclically reduced words of the normal closure of defining words of $\mathcal{P}$ if the group, defined by the presentation $\mathcal{P}$, is trivial. We generalize this result by proving it for every group presentation $\mathcal{P}$ with an obvious exception. We also introduce a new, "cyclic", version of the AndrewsCurtis conjecture and show that the original Andrews-Curtis conjecture with stabilizations is equivalent to its cyclic version.
\end{abstract}

\section{Two THEOREMS}

Let a group $\mathcal{G}$ be defined by a presentation $\mathcal{P}$ in terms of generators and defining relations

$$
\mathcal{P}=\left\langle a_{1}, \ldots, a_{m} \| R=1, R \in \mathcal{R}\right\rangle,
$$

where $\mathcal{R}$ is a set of words in the alphabet $\mathcal{A}^{ \pm 1}=\left\{a_{1}^{ \pm 1}, \ldots, a_{m}^{ \pm 1}\right\}$, whose elements $R \in \mathcal{R}$ are assumed to be cyclically reduced words and are called defining words of G. Recall that this means that $\mathcal{G}=F(\mathcal{A}) / N(\mathcal{R})$ is the quotient group of the free group $F(\mathcal{A})$ in $\mathcal{A}$ by the normal closure $N(\mathcal{R})$ of $\mathcal{R}$ in $F(\mathcal{A})$.

In [7, Rourke introduced the two operations ${ }^{+},{ }^{\sim}$ over a set $\mathcal{R} \subset F(\mathcal{A})$ of cyclically reduced words so that $\mathcal{R}^{+}$denotes the set of all cyclic permutations of words in $\mathcal{R}$ and $\mathcal{R}^{-1}$, and $\mathcal{R}^{\sim}$ stands for the union of $\mathcal{R}$ and the set of all cyclically reduced words obtained from all possible products $R_{1} R_{2}$, where $R_{1}, R_{2} \in \mathcal{R}$.

Let $\mathcal{R}_{\infty}$ be the $\left({ }^{+}, \sim\right)$-closure of $\mathcal{R}$, that is, $\mathcal{R}_{\infty}$ is the minimal set such that $\mathcal{R} \subseteq \mathcal{R}_{\infty}$ and $\mathcal{R}_{\infty}^{+}=\mathcal{R}_{\infty}, \mathcal{R}_{\infty}{ }^{\sim}=\mathcal{R}_{\infty}$. Clearly, $\mathcal{R}_{\infty}$ can be obtained from $\mathcal{R}$ by iterations of operations,$+ \sim$.

The main result of Rourke's article 7 states that if the group $\mathcal{G}$ is trivial, then $\mathcal{A} \subset \mathcal{R}_{\infty}$, that is, $\mathcal{R}_{\infty}$ is the set of all cyclically reduced words of $F(\mathcal{A})$. This result was reproved by Scarabotti [8]. It is also observed in [7] that, in general, the set $\mathcal{R}_{\infty}$ is "distinctly smaller" than the set $\widehat{N}(\mathcal{R})$ of all cyclically reduced words of the normal closure $N(\mathcal{R})$ of $\mathcal{R}$. For example, if $\mathcal{A}=\{a, b\}$ and $\mathcal{R}=\{a\}$, then

Received by the editors December 28, 2004.

2000 Mathematics Subject Classification. Primary 20F05; Secondary 57M20.

This research was supported in part by NSF grants DMS 00-99612 and DMS 04-00476.

(C)2005 American Mathematical Society 
$\mathcal{R}_{\infty}=\left\{a^{k} \mid k \in \mathbb{Z}\right\} \neq \widehat{N}(\mathcal{R})$. More generally, if there is a letter $a \in \mathcal{A}$ such that $a, a^{-1}$ do not occur in words of $\mathcal{R}$, then, obviously, $\mathcal{R}_{\infty} \neq \widehat{N}(\mathcal{R})$. The first result of this article claims that the existence of such a letter is the only obstruction to the equality $\mathcal{R}_{\infty}=\widehat{N}(\mathcal{R})$.

Theorem 1. Let $F(\mathcal{A})$ be the free group in an alphabet $\mathcal{A}$ and let $\mathcal{R} \subset F(\mathcal{A})$ be a set of cyclically reduced words. Then the Rourke closure $\mathcal{R}_{\infty}$ of $\mathcal{R}$ is the set $\widehat{N}(\mathcal{R})$ of all cyclically reduced words of the normal closure $N(\mathcal{R})$ of $\mathcal{R}$ in $F(\mathcal{A})$ unless there exists a letter $a \in \mathcal{A}$ such that both $a, a^{-1}$ do not occur in words of $\mathcal{R}$.

It is rather interesting to apply the Rourke idea of using only cyclic permutations (in place of arbitrary conjugations) in the context of the Andrews-Curtis conjecture; see [1], 2], 3], [5].

Recall that if $\mathcal{W}=\left(W_{1}, \ldots, W_{p}\right)$ is a $p$-tuple of reduced words in $\mathcal{A}^{ \pm 1}$, then Nielsen operations over $\mathcal{W}$ of types (T1)-(T2) are defined as follows.

(T1) For some $i, W_{i} \rightarrow W_{i}^{-1}$ and $W_{k} \rightarrow W_{k}$ for all $k, k \neq i$.

(T2) For some $i, W_{i} \rightarrow W_{i} W_{j}, j \neq i$, and $W_{k} \rightarrow W_{k}$ for all $k, k \neq i$.

We also consider an operation of the third type (T3) so that

(T3) For some $i, W_{i} \rightarrow S W_{i} S^{-1}$, where $S$ is a word in $\mathcal{A}^{ \pm 1}$, and $W_{k} \rightarrow W_{k}$ for all $k, k \neq i$.

Recall that extended Nielsen operations, termed EN-operations, are those of types (T1)-(T3); see [2].

The Andrews-Curtis conjecture, or AC-conjecture, states that if a balanced presentation

$$
\mathcal{P}=\left\langle a_{1}, \ldots, a_{m} \| R_{1}=1, \ldots, R_{m}=1\right\rangle
$$

defines the trivial group, then the $m$-tuple $\mathcal{R}=\left(R_{1}, \ldots, R_{m}\right)$ can be brought to $\mathcal{A}=\left(a_{1}, \ldots, a_{m}\right)$ by a finite sequence of EN-operations.

Consider a new, "cyclic", version of an EN-operation of type (T3) so that

(CT3) for some $i, W_{i} \rightarrow \bar{W}_{i}$, where $\bar{W}_{i}$ is a cyclic permutation of $W_{i}$, and $W_{k} \rightarrow W_{k}$ for all $k, k \neq i$.

Thus, in place of an arbitrary conjugation of $W_{i}$, we can now use only a cyclic permutation of $W_{i}$. Such redefined operations will be called cyclically extended Nielsen operations, or, briefly, CEN-operations, of types (CT1)-(CT3) (operations of types (CT1)-(CT2) are identical to those of types (T1)-(T2)).

Recall that EN-operations apply to tuples of (reduced) words and, for the cyclic version (as in the definition of Rourke's closure), it is more natural to require that CEN-operations apply to tuples of cyclically reduced words. Hence, when making a CEN-operation of type (CT2), we always cyclically reduce the resulting new word $W_{i} W_{j}$.

Now the cyclic version of the Andrews-Curtis conjecture, or CAC-conjecture, states that if the presentation (2) defines the trivial group, then the $m$-tuple $\mathcal{R}=$ $\left(R_{1}, \ldots, R_{m}\right)$ can be brought to $\mathcal{A}=\left(a_{1}, \ldots, a_{m}\right)$ by a finite sequence of CENoperations.

Recall that there is another, more general, version of the AC-conjecture, or $A C$-conjecture with stabilizations, in which a fourth type of operations, called stabilizations, is allowed. 
(T4) Add (or remove) a new letter $b$ both to the alphabet $\mathcal{A}$ and to the set $\mathcal{R}$ of defining words (when removing, $b$ and $b^{-1}$ must not occur in all other words of $\mathcal{R}$ ).

A stabilization that increases the number of letters in $\mathcal{A}$ is termed positive, otherwise, it is negative.

Quite analogously, we consider the cyclic version of the AC-conjecture with stabilizations, or $C A C$-conjecture with stabilizations, in which we use only cyclic permutations (versus arbitrary conjugations) and cyclically reduce the resulting words after each CEN-operation. Observe that, in general, the CAC-conjecture (with stabilizations) is stronger than the original AC-conjecture (with stabilizations, respectively) in the sense that if the CAC-conjecture (with stabilizations) holds for a presentation $\mathcal{P}$, then the AC-conjecture (with stabilizations, respectively) also holds for this $\mathcal{P}$.

Theorem 2. The original Andrews-Curtis conjecture with stabilizations is equivalent to its cyclic version with stabilizations. More specifically, if (2) is a balanced presentation of the trivial group and the defining words $R_{1}, \ldots, R_{m}$ are cyclically reduced, then the $m$-tuple $\mathcal{R}=\left(R_{1}, \ldots, R_{m}\right)$ can be turned into $\mathcal{A}=\left(a_{1}, \ldots, a_{m}\right)$ by a finite sequence of operations (T1)-(T4) if and only if $\mathcal{R}$ can be turned into $\mathcal{A}$ by a finite sequence of operations (CT1)-(CT3), (T4). In addition, if $\mathcal{R}$ can be transformed into $\mathcal{A}$ by operations (T1)-(T3) and $2 s$ stabilizations, then $\mathcal{R}$ can be transformed into $\mathcal{A}$ by operations (CT1)-(CT3) and $2(m+2 s+1)$ stabilizations.

\section{Proof of Theorem 1}

Since one direction in Theorem 1 is obvious, we will be proving that if every letter $a \in \mathcal{A}$ occurs in words of $\mathcal{R}^{ \pm 1}$, then $\mathcal{R}_{\infty}=\widehat{N}(\mathcal{R})$. If $\mathcal{A}$ has a single letter, then Theorem 1 is obvious and we can assume that $|\mathcal{A}|>1$.

Let $X=Y$ denote the equality of elements of the free group $F(\mathcal{A})$ in $\mathcal{A}$ (also called $\mathcal{A}$-words or just words) and let $X \equiv Y$ stand for the graphical (= letter-byletter) equality of words.

Lemma. Let $B$ be a reduced $\mathcal{A}$-word. Then there exists a word $R \in \mathcal{R}_{\infty}$ such that $R \equiv B C$ with some $C$.

Proof. Pick a letter $a \in \mathcal{A}^{ \pm 1}$ and let $R$ be a word in $\mathcal{R}_{\infty}$ so that $R \equiv a D$ (such a word $R$ can already be found in $\mathcal{R}^{+}$). Note that if $E$ is a reduced word such that $E=D^{3}$, then $a^{3} E \in \mathcal{R}_{\infty}$, in particular, $a^{3} E$ is cyclically reduced.

Now, by induction on the length $|B|$ of a word $B$, we will prove the following modified claim that obviously implies the Lemma: Suppose $B$ is a reduced $\mathcal{A}$-word and $B \equiv b_{1} B_{1} \equiv B_{2} b_{2}$, where $b_{1}, b_{2} \in \mathcal{A}^{ \pm 1}$. Then there exists a word $R \in \mathcal{R}_{\infty}$ such that $R \equiv b_{1} B b_{2} C$. Note that this claim is actually proved above when $|B|=1$. To make the induction step, we assume $|B|>1$ and let $B \equiv b_{1} B_{1} \equiv B_{2} b_{2} \equiv B_{3} b_{3} b_{2}$, where $b_{1}, b_{2}, b_{3} \in \mathcal{A}^{ \pm 1}$. By the induction assumption, there are words $R$ and $S$ in $\mathcal{R}_{\infty}$ for $B_{2}$ and $b_{2}$, respectively, such that $R \equiv b_{1} B_{2} b_{3} C$ and $S \equiv b_{2}^{3} D$. Multiplying cyclic permutations $\bar{R} \equiv b_{3} C b_{1} B_{2}, \bar{S} \equiv b_{2}^{2} D b_{2}$ of $R, S$, we get the word

$$
\bar{R} \bar{S} \equiv b_{3} C b_{1} B_{2} b_{2}^{2} D b_{2} \equiv b_{3} C b_{1} B b_{2} D b_{2}
$$

which is cyclically reduced and hence is in $\mathcal{R}_{\infty}$. Its cyclic permutation $b_{1} B b_{2} D b_{2} b_{3} C$ is a word of the form required for $B$ and the Lemma is proved. 
Recall that a van Kampen (or disk) diagram $\Delta$ over presentation (1) is a planar, connected and simply connected 2-complex that is equipped with a labelling function $\varphi$ from the set of oriented edges of $\Delta$ to $\mathcal{A}^{ \pm 1}$ and has the following two properties (for more details see [4, 6]):

(L1) If $e$ is an oriented edge of $\Delta$, then $\varphi\left(e^{-1}\right)=\varphi(e)^{-1}$.

(L2) If $\Pi$ is a face of $\Delta$, then $\varphi(\partial \Pi)$ is a cyclic permutation of a word in $\mathcal{R} \cup \mathcal{R}^{-1}$.

Now suppose that $W$ is a cyclically reduced nonempty word and $W=1$ in the group $\mathcal{G}$ defined by presentation (1), that is, $W \in \widehat{N}(\mathcal{R})$. Then there is a van Kampen diagram $\Delta=\Delta(W)$ (see [4, 6]) over (11) such that $\varphi(\partial \Delta) \equiv W$, where the boundary $\partial \Delta$ of $\Delta$ is negatively (clockwise) oriented. We will prove that $W \in \mathcal{R}_{\infty}$ by induction on the number $|\Delta(2)|$ of faces in $\Delta$.

As in [4, 6, assume that the boundary $\partial \Pi$ of a face $\Pi$ of $\Delta$ is positively (counterclockwise) oriented. Pick a face $\Pi$ in $\Delta$ so that there is an edge $e \in \partial \Pi$ with $e^{-1} \in \partial \Delta$. Denote $\partial \Pi=e u, \partial \Delta=e^{-1} d$; see Figure 1 .

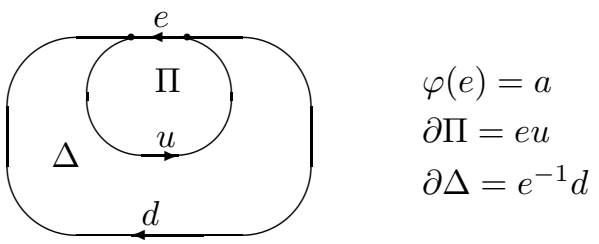

\section{FiguRe 1}

Taking $e$ and $\Pi$ out of $\Delta$, we will get a diagram $\Delta_{1}$ such that $\partial \Delta_{1}=d u$ and $\left|\Delta_{1}(2)\right|=|\Delta(2)|-1$.

If $\varphi\left(\partial \Delta_{1}\right) \equiv \varphi(d) \varphi(u)=1$ in $F(\mathcal{A})$, then $\varphi(\partial \Delta) \equiv \varphi(\partial \Pi)^{-1} \equiv W$. Hence, $W \in \mathcal{R}_{\infty}$, as required.

Now assume that $\varphi\left(\partial \Delta_{1}\right) \neq 1$ in $F(\mathcal{A})$. Note that the words $\varphi(d), \varphi(u)$ are subwords of cyclically reduced words $W, \varphi(\partial \Pi)$, respectively, and hence $\varphi(d), \varphi(u)$ are reduced. Since the product $\varphi(d) \varphi(u)$ need not be (cyclically) reduced, it follows that the words $D \equiv \varphi(d)^{-1}$ and $U \equiv \varphi(u)$ can be represented in one of the following three forms (F1)-(F3).

(F1) $D \equiv B D_{1} E$ and $U \equiv B U_{1} E$, where $D_{1} U_{1}^{-1}$ is a nonempty cyclically reduced word (some of $B, D_{1}, U_{1}, E$ may be empty).

(F2) $D \equiv B E$ and $U \equiv B S U_{0} S^{-1} E$, where $U_{0}$ is a nonempty cyclically reduced word (some of $B, S, E$ may be empty).

(F3) $D \equiv B S D_{0} S^{-1} E$ and $U \equiv B E$, where $D_{0}$ is a nonempty cyclically reduced word (some of $B, S, E$ may be empty).

Observe that there is a diagram $\Delta_{0}$ such that $\left|\Delta_{0}(2)\right| \leq|\Delta(2)|-1$ and $\varphi\left(\partial \Delta_{0}\right) \equiv$ $D_{1} U_{1}^{-1}$ in Case (F1) or $\varphi\left(\partial \Delta_{0}\right) \equiv U_{0}$ in Case (F2) or $\varphi\left(\partial \Delta_{0}\right) \equiv D_{0}$ in Case (F3).

Since $\varphi\left(\partial \Delta_{0}\right)$ is cyclically reduced, it follows from the induction assumption that $\phi\left(\partial \Delta_{0}\right) \in \mathcal{R}_{\infty}$. Denote $\varphi(e)=a$, where $a \in \mathcal{A}^{ \pm 1}$. In Cases (F1)-(F2), we can see that $W$ is a cyclic permutation of the product of some cyclic permutations of $\phi\left(\partial \Delta_{0}\right)$ and $\varphi(\partial \Pi)^{-1}$; see Figure 2(a) in Case (F1) and Figure 2(b) in Case (F2). 


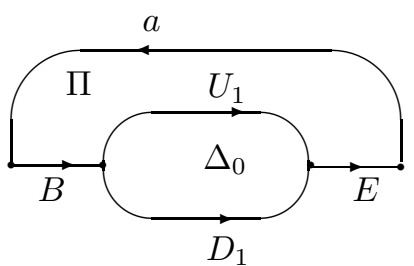

Figure 2(a)

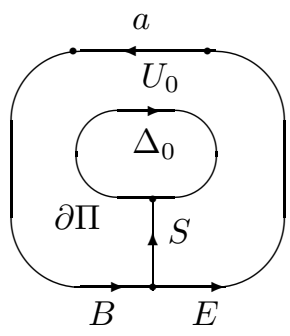

Figure 2(b)

In Case (F3), $W$ is a cyclic permutation of the cyclically reduced product of words $\varphi(\partial \Pi)^{-1} \equiv B^{-1} a^{-1} E^{-1}$ and $S D_{0}^{-1} S^{-1}$; see Figure $3(\mathrm{a})$.

It follows from the Lemma, applied to a word of the form $x S y$, where $x, y \in$ $\mathcal{A}^{ \pm 1}$ are letters such that $x E, D_{0} y$ are reduced (recall $|\mathcal{A}|>1$ ), that there is a word $R \in \mathcal{R}_{\infty}$ such that $R \equiv S T$ for some $T$ of the form $T \equiv y T_{0} x$ and the word $E a B S D_{0} T$ is cyclically reduced. Since the word $E a B S D_{0} T$ is the product of cyclic permutations of $\varphi(\partial \Pi)$ and $T S \varphi\left(\partial \Delta_{0}\right)^{-1}$, and $T S \varphi\left(\partial \Delta_{0}\right)^{-1}$ in its turn is the product of cyclic permutations of $R \equiv S T$ and $D_{0} \equiv \varphi\left(\partial \Delta_{0}\right)^{-1}$ (see Figure 3(b)), it follows that $\operatorname{EaBS} D_{0} T \in \mathcal{R}_{\infty}$. Now we observe that $W^{-1}$ is a cyclic permutation of the product of some cyclic permutations of $E a B S D_{0} T$ and $R^{-1} \equiv T^{-1} S^{-1}$. Thus, $W \in \mathcal{R}_{\infty}$ and Theorem 1 is proved.

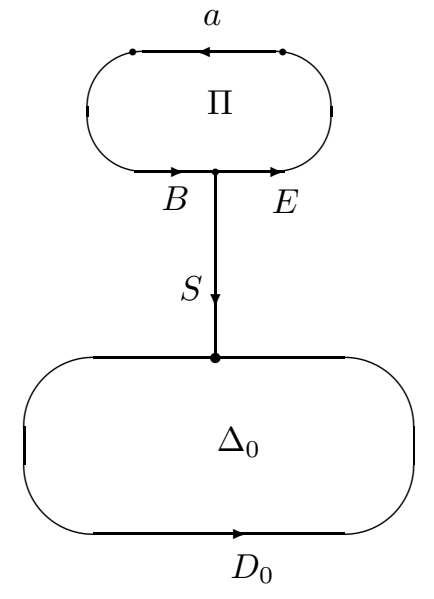

FiguRe 3(a)

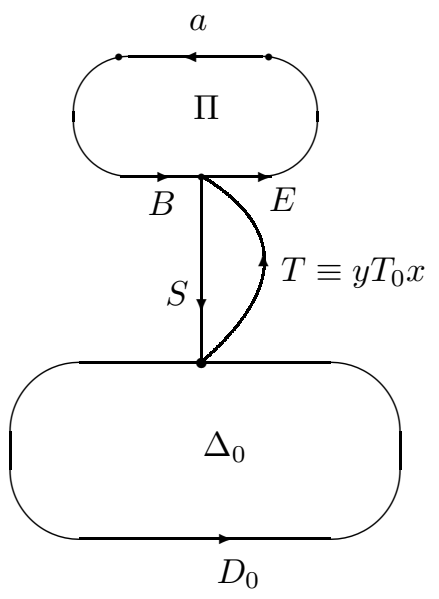

FiguRE 3(b)

\section{Proof of Theorem 2}

Recall that the input and output of an EN-operation are tuples of (reduced) words while those of a CEN-operation are tuples of cyclically reduced words. Since CEN-operations are more restrictive and a cyclic permutation of a word can be realized by an EN-operation of type (T3), it follows that we only need to show that if $\mathcal{R}=\left(R_{1}, \ldots, R_{m}\right)$ can be turned into an $\mathcal{A}=\left(a_{1}, \ldots, a_{m}\right)$ by a sequence of ENoperations and $2 s$ stabilizations, then $\mathcal{R}$ can also be turned into $\mathcal{A}$ by a sequence of CEN-operations and at most $2(m+2 s+1)$ stabilizations. 
Furthermore, it is clear that, in the process of getting $\mathcal{A}$ from $\mathcal{R}$ by EN-operations and $2 s$ stabilizations, we can do all $s$ positive stabilizations in the very beginning and all $s$ negative stabilizations in the very end. Therefore, we can avoid stabilizations altogether and assume that the $(m+s)$-tuple $(\mathcal{R}, \mathcal{B})=\left(R_{1}, \ldots, R_{m}, b_{1}, \ldots, b_{s}\right)$, where $b_{1}, \ldots, b_{s}$ are all new letters that were introduced by $s$ positive stabilizations, can be turned into the $(m+s)$-tuple $(\mathcal{A}, \mathcal{B})=\left(a_{1}, \ldots, a_{m}, b_{1}, \ldots, b_{s}\right)$ by a sequence of EN-operations $\sigma_{1}, \sigma_{2}, \ldots, \sigma_{\ell}$ of types (T1)-(T3).

Now, with the original $(m+s)$-tuple $\mathcal{W}(0)=(\mathcal{R}, \mathcal{B})$, we associate a $(2 m+2 s+1)$ tuple

$$
\begin{aligned}
& \mathcal{U}(0)=\left(R_{1}, \ldots, R_{m}, b_{1}, \ldots, b_{s},\right. \\
& \left.\qquad x_{1} R_{1}, \ldots, x_{m} R_{m}, x_{m+1} b_{1}, \ldots, x_{m+s} b_{s}, y\right) .
\end{aligned}
$$

Clearly, $\mathcal{U}(0)$ can be obtained from $\mathcal{R}$ by $m+2 s+1$ positive stabilizations and by CEN-operations (CT1)-(CT2).

Let

$$
\mathcal{W}(k)=\left(W_{1}, \ldots, W_{m+s}\right)
$$

be the $(m+s)$-tuple obtained from $\mathcal{W}(0)$ by the first $k \geq 0$ EN-operations $\sigma_{1}, \ldots, \sigma_{k}$. Now, by induction on $k \geq 0$ (the base $k=0$ is trivial), assume that a $(2 m+2 s+1)$-tuple $\mathcal{U}(k)=\left(U_{1}, \ldots, U_{2 m+2 s+1}\right)$ of cyclically reduced words is already constructed so that $U_{i}$ is freely conjugate (that is, conjugate in a free group) to $W_{i}$ for $1 \leq i \leq m+s$ and, for $i>m+s$, we have

$$
\begin{aligned}
& U_{m+s+1} \equiv x_{1} R_{1}, \ldots, U_{2 m+s} \equiv x_{m} R_{m}, U_{2 m+s+1} \equiv x_{m+1} b_{1}, \ldots, \\
& U_{2 m+2 s} \equiv x_{m+s} b_{s}, U_{2 m+2 s+1} \equiv y \text {. }
\end{aligned}
$$

Let $\mathcal{W}(k+1)$ be obtained from $\mathcal{W}(k)$ by the next EN-operation $\sigma_{k+1}$. Our goal is to find a sequence of CEN-operations that would turn $\mathcal{U}(k)$ into $\mathcal{U}(k+1)$, where $\mathcal{U}(k+1)$ corresponds to $\mathcal{W}(k+1)$ in the same fashion as $\mathcal{U}(k)$ corresponds to $\mathcal{W}(k)$.

If $\sigma_{k+1}$ has type (T1), that is, $W_{i} \rightarrow W_{i}^{-1}$, then we make a similar CENoperation over $\mathcal{U}(k)$ which is $U_{i} \rightarrow U_{i}^{-1}$. If $\sigma_{k+1}$ has type (T3), that is, $W_{i} \rightarrow$ $S W_{i} S^{-1}$, then no change is needed and $\mathcal{U}(k+1)=\mathcal{U}(k)$.

Now consider the main case when $\sigma_{k+1}$ has type (T2) and $W_{i} \rightarrow W_{i} W_{j}, j \neq i$, $1 \leq i, j \leq m+s$. Note that for some reduced word $C$ in the alphabet $(\mathcal{A} \cup \mathcal{B})^{ \pm 1}=$ $\left\{a_{1}^{ \pm 1}, \ldots, a_{m}^{ \pm 1}, b_{1}^{ \pm 1}, \ldots, b_{s}^{ \pm 1}\right\}$, the word $U_{i} C U_{j} C^{-1}$ is freely conjugate to $W_{i} W_{j}$.

Let $P_{i}$ denote a cyclically reduced word which is freely conjugate to $U_{i} C U_{j} C^{-1}$. We will construct a sequence of CEN-operations that turn the word $U_{i}$ into $P_{i}$ and preserve all other components of $\mathcal{U}(k)$. This new $(2 m+2 s+1)$-tuple will be the desired $\mathcal{U}(k+1)$.

Let $C \equiv c_{1} \ldots c_{d}$, where $c_{1}, \ldots, c_{d}$ are letters in $(\mathcal{A} \cup \mathcal{B})^{ \pm 1}$, and let $Q_{1}, \ldots, Q_{d}$ be cyclic permutations of some words among $U_{m+s+1}^{ \pm 1}, \ldots, U_{2 m+2 s}^{ \pm 1}$ (see (14)) such that $Q_{1} \equiv c_{1} T_{1}, \ldots, Q_{d} \equiv c_{d} T_{d}$.

Consider a sequence of CEN-operations (CT1)-(CT3) so that

$$
\begin{aligned}
& U_{i} \rightarrow y U_{i} y \rightarrow y U_{i} y c_{1} T_{1} \rightarrow T_{1} y U_{i} y c_{1} \\
& \rightarrow y T_{2} y T_{1} y U_{i} y c_{1} c_{2} \rightarrow \cdots T_{1} y U_{i} y c_{1} c_{2} T_{2} \\
& \rightarrow \cdots \rightarrow y T_{d} y T_{d-1} \ldots y T_{1} y U_{i} y c_{1} \ldots c_{d} y U_{j} \\
& \rightarrow U_{i} y c_{1} \ldots c_{d} y U_{j} y T_{d} y T_{d-1} \ldots y T_{1} y \equiv U_{i, 1}
\end{aligned}
$$

and all other components of $\mathcal{U}(k)$ are preserved. Now we will use more CENoperations to turn the subwords $T_{d}, \ldots, T_{1}$ of $U_{i, 1}$ into $c_{d}^{-1}, \ldots, c_{1}^{-1}$, respectively. 
Doing this transforms the word $U_{i, 1}$ into

$$
U_{i, 2} \equiv U_{i} y c_{1} \ldots c_{d} y U_{j} y c_{d}^{-1} y c_{d-1}^{-1} \ldots y c_{1}^{-1} y .
$$

Using more CEN-operations of types (CT1)-(CT3) (the $j$ index in CEN-operations of type (CT2) is now $2 m+2 s+1$ ), we delete all occurrences of letters $y^{ \pm 1}$ in $U_{i, 2}$. Doing this turns the word $U_{i, 2}$ into a cyclic permutation of the word $P_{i}$ and an application of (CT3) finally yields the desired $(2 m+2 s+1)$-tuple $\mathcal{U}(k+1)$ whose $i$ th component is $P_{i}$ and all other components are those of $\mathcal{U}(k)$.

Thus, by induction, it is proved that for every $k \geq 0$ there is a sequence of CENoperations that turn the tuple $\mathcal{U}(0)$ (see (3) ) into a $(2 m+2 s+1)$-tuple $\mathfrak{U}(k)=$ $\left(U_{1}, \ldots, U_{2 m+2 s+1}\right)$ such that $U_{m+s+1}, \ldots, U_{2 m+2 s+1}$ are given by formulas (4) and $U_{1}, \ldots, U_{m+s}$ are cyclically reduced words freely conjugate to $W_{1}, \ldots, W_{m+s}$, respectively, where $\mathcal{W}(k)=\left(W_{1}, \ldots, W_{m+s}\right)$ is obtained from $\mathcal{W}(0)$ by the first $k$ EN-operations $\sigma_{1}, \ldots, \sigma_{k}$. Since $\mathcal{W}(\ell)=\left(a_{1}, \ldots, a_{m}, b_{1}, \ldots, b_{s}\right)$, it follows that

$$
\mathcal{U}(\ell)=\left(a_{1}, \ldots, a_{m}, b_{1}, \ldots, b_{s}, U_{m+s+1}, \ldots, U_{2 m+2 s+1}\right) .
$$

Now we can use obvious CEN-operations and $m+2 s+1$ negative stabilizations to change $\mathcal{U}(\ell)$ into $\left(a_{1}, \ldots, a_{m}\right)$. Theorem 2 is proved.

\section{REFERENCES}

[1] J.J. Andrews and M.L. Curtis, Free groups and handlebodies, Proc. Amer. Math. Soc. 16(1965), 192-195. MR0173241 (30:3454)

[2] J.J. Andrews and M.L. Curtis, Extended Nielsen operations in free groups, Amer. Math. Monthly 73(1966), 21-28. MR0195928 (33:4124)

[3] R.G. Burns and O. Macedonska, Balanced presentations of the trivial group, Bull. London Math. Soc. 25(1993), 513-526. MR1245076 (94i:20050)

[4] S.V. Ivanov, The free Burnside groups of sufficiently large exponents, Internat. J. Algebra Comp. 4(1994), 1-308. MR 1283947 (95h:20051)

[5] A.D. Myasnikov, A.G. Myasnikov, and V. Shpilrain, On the Andrews-Curtis equivalence, Contemp. Math. 296(2002), 183-198. MR1921712 (2003g:20053)

[6] A.Yu. Ol'shanskii, Geometry of defining relations in groups, Nauka, Moscow, 1989; English translation: Math. and Its Appl., Soviet series 70, Kluwer Acad. Publ., 1991. MR.1024791 (91i:20035)

[7] C.P. Rourke, Presentations and the trivial group, in Topology of low-dimensional manifolds, Lecture Notes in Math., vol. 722, Springer-Verlag, 1979, pp. 134-143. MR0547460 (81a:57001)

[8] F. Scarabotti, On the presentations of the trivial group, J. Group Theory 2(1999), 319-327. MR.1696318(2000e:20058)

Department of Mathematics, University of Illinois, Urbana, Illinois 61801

E-mail address: ivanov@math.uiuc.edu 